\title{
TOPOSES ARE COHOMOLOGICALLY EQUIVALENT TO SPACES
}

\author{
By A. Joyal and I. MoERdIJK ${ }^{1}$
}

This purpose of this paper is to prove that for every Grothendieck topos $\mathscr{E}$ there exist a space $X$ and a covering $\varphi: X \rightarrow \mathscr{E}$ which induces an isomorphism in cohomology

$$
H^{n}(\mathscr{E}, A) \stackrel{\sim}{\rightarrow} H^{n}\left(X, \varphi^{*} A\right) \quad(n \geq 0)
$$

for any abelian group $A$ in $\mathscr{E}$. Moreover for $n=1$ this is also true for nonabelian $A$. This implies, by a result of Artin and Mazur, that $\varphi$ induces an isomorphism of etale homotopy groups.

1. Construction of the cover. Let $\mathscr{E}$ be a Grothendieck topos, and let $G$ be an object of $\mathscr{E}$. $\operatorname{En}(G)$ is the space (in this paper 'space' means space in the sense of [JT], chapter IV, unless explicitly said otherwise) of infinite-to-one partial enumerations of $G$; in other words, $\operatorname{En}(G)$ is characterized by the property that for any map $f: \mathscr{F} \rightarrow \mathscr{E}$ of toposes, the points of the induced space $f^{\#}(\operatorname{En}(G))$ in $\mathscr{F}_{F}$ correspond to diagrams $\mathbf{N}<-<\longrightarrow f^{*} G$ in $\mathscr{F}$ with the property that for any $n \in \mathbf{N}, U-$ $\{0, \ldots, n\} \rightarrow f^{*} G$ is still epi. We write $\mathscr{E}[\operatorname{En}(G)]$ for the category of sheaves in $\mathscr{E}$ on the space $\operatorname{En}(G)$, and $\varphi: \mathscr{E}(\operatorname{En}(G)] \rightarrow \mathscr{E}$ for the corresponding geometric morphism. The properties of the space $\operatorname{En}(G)$ and the map $\varphi$ were extensively discussed in [JM]. For the present purpose, we recall the following basic facts. First of all, for a suitable object $G$ of $\mathscr{E}, \mathscr{E}[\operatorname{En}(G)]$ is equivalent to the topos $\operatorname{Sh}\left(X_{\mathscr{E}}\right)$ of sheaves on a space $X_{\mathscr{E}}$ in Sets, so that $\varphi$ corresponds to a cover

$$
\varphi: \operatorname{Sh}\left(X_{\mathscr{E}}\right) \rightarrow \mathscr{E} .
$$

Manuscript received 6 November 1988.

${ }^{1}$ Supported by a Huygens Fellowship of the NWO.

American Journal of Mathematics 112 (1990), 87-95. 
This geometric morphism is connected and locally connected; in partic-

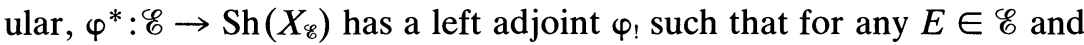
$S \in \operatorname{Sh}\left(X_{\mathscr{E}}\right)$,

$$
\varphi_{!}\left(\varphi^{*}(E) \times S\right) \cong E \times \varphi_{!}(S)
$$

For any $G$ in $\mathscr{E}$, there exists a surjective geometric morphism $p: \mathscr{B} \rightarrow \mathscr{E}$ where $\mathscr{B}$ is the category of sheaves on a complete Boolean algebra (Barr's theorem, [B]), such that $p^{*} G$ is countable (cf. [JT]). $\mathscr{B}$ is a model of set theory, and the induced space $\operatorname{En}\left(p^{*} G\right) \cong p^{\#}(\operatorname{En}(G))$ in $\mathscr{B}$ has enough points, i.e. is an ordinary topological space, which can be described as follows: the points of $\operatorname{En}\left(p^{\#}(G)\right)$ are functions $\alpha: U \rightarrow$ $p^{*} G$ with $U \subset \mathbf{N}$ and $\alpha^{-1}(g)$ infinite for all $g \in p^{*}(G)$; the basic open sets are the sets of the form $V_{u}=\{\alpha \mid \forall i \in \operatorname{domain}(u): i \in U$ and $\alpha(i)$ $=u(i)\}$, where $u$ ranges over all functions $u: K \rightarrow p^{*} G$ defined on a finite set $K \subset \mathbf{N}$. It is not difficult to prove that each basic open set $V_{u}$ (in particular, the space itself, $V_{\phi}$ ) is contractible ([JM]).

2. Relative Čech cohomology. In this section, let $Y$ be a space in a topos $\mathscr{E}$. One can define the relative Čech cohomology groups of $Y$ with coefficients in an abelian group object in $\mathscr{E}[Y]$, i.e. a sheaf (or in fact, just a presheaf) of abelian groups on $Y$ in $\mathscr{E}$,

$$
\check{H}_{\mathscr{g}}^{n}(Y, A)
$$

These cohomology groups are group objects in $\mathscr{E}$. Their construction is completely parallel to the usual construction of the Čech cohomology groups of a topological space; indeed, the latter construction immediately translates to the context of a space in a topos $\mathscr{E}$, by viewing $\mathscr{E}$ as a universe for (constructive) set theory (cf. $[\mathrm{BJ}]$ ).

More explicitly, let $S \in \mathscr{E}$ and let $\mathcal{U}: S \rightarrow \mathcal{O}(Y)$ be an open cover of $Y$ indexed by $S$. Let $A$ be a (pre)sheaf of abelian groups on $Y$ in $\mathscr{E}$; so $A$ is given by a map $A \rightarrow O(Y)$ in $\mathscr{E}$ equipped with the structure of a (pre)sheaf. Let

$$
\mathcal{U}_{p}: S_{p}=S \underset{-p+1-}{\times \cdots} \stackrel{\varkappa^{p+1}}{\longrightarrow} \mathcal{O}(Y)^{p+1} \stackrel{\wedge}{\longrightarrow} \mathcal{O}(Y)
$$

be the map in $\mathscr{E}$ obtained from $\mathscr{U}$ by intersection in $Y$, and let 


$$
C^{p}(\mathcal{U}, A)=\prod_{S_{p}}\left(A \underset{\odot(Y)}{\times} S_{p} \rightarrow S_{p}\right)
$$

where $\Pi_{S_{p}}: \mathscr{E} / S_{p} \rightarrow \mathscr{E}$ is the right adjoint of the functor $S_{p}{ }^{*}: \mathscr{E} \rightarrow \mathscr{E} / S_{p}$ (cf. [J], p. 36). The $C^{p}(U, A), p \geq 0$, give a cochain complex $C^{0}(U, A)$ $\rightarrow C^{1}(U, A) \rightarrow \cdots$ in the usual way, with the differential defined via alternating sums. The cohomology groups of this complex are denoted by $H_{\mathscr{\varepsilon}}^{n}(U, A)$. One may now take the colimit of these groups over the internal diagram in $\mathscr{E}$ of all open covers of $O(Y)$ (so this involves internal covers of $Y$ in $\mathscr{E} / E$ for arbitrary $E$ !), and obtain the relative Čech cohomology groups

$$
\check{H}_{\mathscr{E}}^{n}(Y, A)=\lim _{\rightarrow \mathcal{U}} H_{\mathscr{E}}^{p}(U, A) \quad(p \geq 0)
$$

Straightforward modifications of the standard argument show that these cohomology groups have the usual properties. For instance, if we write $\varphi: \mathscr{E}[Y] \rightarrow \mathscr{E}$ for the canonical geometric morphism and $e_{E}: \mathscr{E} / E \rightarrow \mathscr{E}$ for the geometric morphism given by $e_{E}^{*}=E^{*}=\left(X \mapsto X \times E \stackrel{\pi_{2}}{\rightarrow} E\right)$, then for any open cover $\mathcal{U}$ of $e_{E}^{*}(Y)$ in $\mathscr{E} / E$,

$$
H_{\mathscr{E} / E}^{0}\left(U, e_{E}^{*}(A)\right) \cong e_{E}^{*} \varphi_{*} A,
$$

where $E$ is any object of $\mathscr{E}$; hence

$$
H_{\mathscr{8}}^{0}(Y, A) \cong \varphi_{*} A
$$

And for an injective object I of the category $\underline{\mathrm{Ab}} \mathscr{E}[Y]$ of abelian sheaves on $Y$ in $\mathscr{E}$,

$$
H_{\mathscr{E} / E}^{n}\left(U, e_{E}^{*} I\right)=0 \quad(n>0)
$$

for any $E$ in $\mathscr{E}$ and any open cover $\mathcal{U}$ of $e_{E}^{\#}(Y)$ in $\mathscr{E} / E$, so

$$
\check{H}_{\mathscr{E}}^{n}(Y, I)=0 \quad(n>0)
$$

3. A relative Cartan-Leray spectral sequence. As before, let $Y$ be a space in a topos $\mathscr{E}$, and let $\varphi: \mathscr{E}[Y] \rightarrow \mathscr{E}$ be the corresponding 
geometric morphism. $\mathscr{E}[Y]$ is a subtopos of the topos $\mathscr{E} O(Y)^{o p}$ of presheaves on $\mathscr{O}(Y)$ in $\mathscr{E}$, and we write $\left.i: \mathscr{E}[Y] \hookrightarrow \mathscr{E}^{\mathscr{O}(Y)}\right)^{o p}$ for the inclusion. The following is a relative version of SGA4, exp V, p. 24.

Lemma 1. For any abelian group $A$ in $\mathscr{E}[Y]$, there exists a spectral sequence

$$
E_{2}^{p, q}=\check{H}_{\mathscr{\varepsilon}}^{p}\left(Y, R^{q} i_{*}(A)\right) \Rightarrow R^{p+q} \varphi_{*}(A)
$$

Proof. Let $0 \rightarrow A \rightarrow I^{\circ}$ be an injective resolution of $A$ in $\underline{\mathrm{Ab}} \mathscr{E}[Y]$. For an open cover $U$ of $Y$ in $\mathscr{E}$, one has a double complex of abelian groups $C^{p, q}(U)=C^{p}\left(U, I^{q}\right)$ (cf. (3)). By (5) and (7) above, the cohomology of the total complex is $H^{n} H^{0}\left(C^{* *}(U)\right)=R^{n} \varphi_{*}(A)$, so we obtain a spectral sequence

$$
E_{2}^{p, q}(U)=H^{p} H^{q}\left(C^{* *}(U)\right)=H_{\varepsilon}^{p}\left(u, R^{q} i_{*} A\right) \Rightarrow R^{p+q} \varphi_{*}(A)
$$

in the standard way ([G]). The same applies to open covers of $e_{E}^{\#}(Y)$ in $\mathscr{E} / E$ for any object $E$ of $\mathscr{E}$, so by taking the internal colimit in $\mathscr{E}$ over all open covers of $Y$, we obtain a spectral sequence as stated in the lemma.

Now let $\mathbf{B} \subset \mathcal{O}(Y)$ be a basis for $Y$ in $\mathscr{E}$ which is closed under binary meets. Call $\mathbf{B} A$-acyclic if for every morphism $B: E \rightarrow \mathbf{B}$ in $\mathscr{E}$,

$$
\check{H}_{\mathscr{E} / E}^{p}(B, A \mid B)=0 . \quad(q>0)
$$

In (10), $B$ stands for the open subspace of $e_{E}^{\#}(Y)$ determined by the given morphism $B: E \rightarrow \mathbf{B} \subset \mathcal{O}(Y)$, and $A \mid B \in \underline{\mathrm{Ab}}((\mathscr{E} / E)[B])$ is the sheaf induced by $A$.

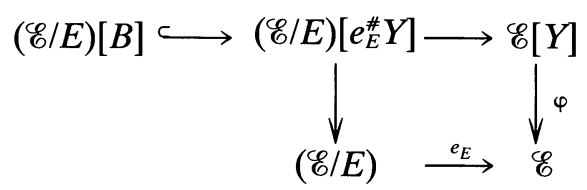

Lemma 2. If $\mathbf{B}$ is an A-acyclic basis for $Y$ as above, then $\check{H}_{\dot{g}}^{p}(Y, A)$ $\cong R^{p} \varphi_{*} A$, for all $p \geq 0$. 
Proof. We show by induction on $n$ that $E_{2}^{p, q}=0$ for all $p$ and all $q$ with $0<q<n$, in the spectral sequence of Lemma 1. Suppose this holds for $n$. Then (cf. [CE], p. 328) $\check{H}_{\mathscr{g}}^{i}(Y, A)=R^{i} \varphi_{*} A$ for $i<n$, and there is an exact sequence $0 \rightarrow \check{H}_{\mathscr{E}}^{n}(Y, A) \rightarrow R^{i} \varphi_{*} A \rightarrow E_{2}^{0, n} \rightarrow$ $\check{H}_{\mathscr{E}}^{n+1}(Y, A) \rightarrow R^{n+1} \varphi_{*} A$. But $E_{2}^{0, n}=\check{H}_{\mathscr{\&}}^{0}\left(Y, R^{n} i_{*} A\right) \longrightarrow \varphi_{*} i^{*} R^{n} i_{*}(A)$ $=\varphi_{*} R^{n} i^{*} i_{*}(A)=\varphi_{*}(0)=0(n>0)$, so $\check{H}_{\mathscr{E}}^{n}(Y, A) \cong R^{n} \varphi_{*} A$. Applying this argument not to $Y$, but to any open subspace $B$ (for any morphism $B: E \rightarrow \mathbf{B}$, cf the diagram (11)), our assumption on $\mathbf{B}$ gives that $R^{n} i_{*}(A)$ $\mid \mathbf{B}=0$, where $(-) \mid \mathbf{B}$ denotes the restriction functor $\mathscr{E} O(X)^{o p} \rightarrow \mathscr{E}^{\mathbf{B}^{o p}}$. Thus if in the spectral sequence (9) above, $U$ is a cover consisting of basic opens from $\mathbf{B}$, then $E_{2}^{p, n}(u)=H_{E}^{p}(u, 0)=0$ for all $p$. Since such covers consisting of basic opens are cofinal in the internal system of all covers, it follows by passing to the colimit that $E_{2}^{p, n}=0$ (all $p$ ) in the spectral sequence of Lemma 1 . So the inductive statement in the beginning of the proof holds for $n+1$, and Lemma 2 is proved.

Remark. Let $Y$ be a space in $\mathscr{E}$, as above. Recall (see [JT]) that an open $U \subset Y$ is called surjective if it holds in $\mathscr{E}$ that every cover of $U$ is inhabited. If $A$ is a sheaf on $Y$ and $\left\{U_{\alpha}: \alpha \in \mathscr{A}\right\}$ is a family of opens, then $\Pi\left\{A\left(U_{\alpha}\right) \mid \alpha \in \mathscr{A}\right\} \cong \Pi\left\{A\left(U_{\alpha}\right) \mid \alpha \in \mathscr{A}, U_{\alpha}\right.$ surjective $\}$ (where $\Pi$ is the internal product $\mathscr{E} / \mathscr{A} \rightarrow \mathscr{E}$, as in Section 2). This is analogous to the fact that for $\mathscr{E}=$ Sets, $A(U)=\left\{{ }^{*}\right\}$ if the empty set covers $U$. Therefore in Lemma 2 it is enough to assume that $\mathbf{B}$ is closed under surjective binary meets (i.e. $B \wedge B^{\prime} \in \mathbf{B}$ whenever $B$ and $B^{\prime} \in \mathbf{B}$ and $B \wedge B^{\prime}$ is surjective), since by this isomorphism, surjective intersections are the only ones that need to be considered in the complexes $C^{p, q}(U)$.

4. The main theorem. Let $\mathscr{E}$ be a Grothendieck topos, and let $X_{\mathscr{E}}$ be the space constructed in Section 1. In the following theorem, $H^{q}\left(X_{\mathscr{E}},-\right)$ denotes the sheaf cohomology of $X_{\mathscr{E}}$.

TheOREM. The geometric morphism $\varphi: \operatorname{Sh}\left(X_{\mathscr{E}}\right) \rightarrow \mathscr{E}$ has the property that for any abelian group $A$ in $\mathscr{E}, R^{q} \varphi_{*}\left(\varphi^{*} A\right)=0$ for $q>0$ (for $\left.q=0, R^{q} \varphi_{*}\left(\varphi^{*} A\right) \cong A\right)$; consequently, $\varphi$ induces an isomorphism

$$
H^{q}(\mathscr{E}, A) \stackrel{\sim}{\rightarrow} H^{q}\left(X_{\mathscr{E}}, \varphi^{*} A\right)
$$

for each $q \geq 0$. 
Proof. The second statement follows from the first by the Leray spectral sequence (SGA4, exp V, p. 35). The first statement is a special case (by construction of $X_{\mathscr{E}}$ ) of the general fact that for any object $G$ in $\mathscr{E}$, the corresponding geometric morphism $\varphi: \mathscr{E}[\operatorname{En}(G)] \rightarrow \mathscr{E}$ induces isomorphisms $H^{q}(\mathscr{E}, A) \stackrel{\sim}{\rightarrow} H^{q}\left(\mathscr{E}[E(G)], \varphi^{*} A\right)$, for any abelian group $A$ in $\mathscr{E}$ and any $q \geq 0$. Let $\mathbf{B}$ be the basis consisting of opens of the form $V_{u}$ ( $u$ a finite partial function from $\mathbf{N}$ to $G$, cf. [JM]). $\operatorname{En}(G)=V_{\phi} \in$ $\mathbf{B}$, and $V_{u} \wedge V_{w}$ is surjective iff $u$ and $w$ are compatible finite functions, and in that case $V_{u} \wedge V_{w}=V_{u \cup w}$, so $\mathbf{B}$ is closed under surjective finite meets (cf. the remark in Section 3).

We will show that for any injective object $I$ of $\underline{A b}(\mathscr{E})$ and any $q>0$

$$
R^{q} \varphi_{*}\left(\varphi^{*} I\right)=0
$$

This is enough, because $\varphi$ is connected, i.e. $\varphi_{*} \varphi^{*} \cong \mathrm{id}$, and (12) says that $\varphi^{*}$ maps injectives to $\varphi_{*}$-acyclic objects, so there is a spectral sequence ([G]) for the composition $\varphi^{*} \circ \varphi_{*}, E_{2}^{p, q}=\left(R^{p} \varphi_{*}\right)\left(R^{q} \varphi^{*}\right) A \Rightarrow$ $R^{p+q}\left(\varphi_{*} \varphi^{*}\right) A ; \varphi^{*}$ is exact and $\varphi_{*} \varphi^{*} \cong$ id, so $E_{2}^{p, q}=0$ for $q>0$ and $E_{2}^{p, 0}=R^{p}(\mathrm{id})(A)=0$ for $p>0$. Thus $R^{p} \varphi_{*}\left(\varphi^{*} A\right)=0$ for $p>0$.

To prove (12), let $I$ be an injective in $\underline{\mathrm{Ab}} \mathscr{E}$, and let $U$ be an open cover of $\operatorname{En}(G)$ by basic opens, say $U: S \rightarrow \mathbf{B} \subset \mathcal{O}(Y)$ as in Section 2 . Let us consider the nerve $N(U)$ of $U$. This is the simplicial complex in $\mathscr{E}$ defined as follows: $S$. $=\left(S_{p}, p \geq 0\right)$ is a simplicial complex in $\mathscr{E}$, with as face $d_{i}: S_{p} \rightarrow S_{p-1}$ the projection $S^{p+1} \rightarrow S^{p}$ which deletes the $i$-th coordinate. The morphism $U_{p}: S_{p} \rightarrow \mathbf{B} \subset \mathcal{O}(Y)$ can be viewed as an $S_{p^{-}}$ indexed sum of subobjects of the terminal object 1 of $\mathscr{E}[Y]$, and we write $\Sigma_{S_{p}} u_{p}$ for their internal sum. Then

$$
N_{p}(\mathcal{U})=\varphi !\left(\sum_{s_{p}} \mathcal{U}_{p}\right)
$$

and the faces and degeneracies of $S$. give $N .(U)$ the structure of a simplicial complex over $\mathscr{E}$. Moreover,

$$
C^{p}\left(U, \varphi^{*} I\right) \cong I^{N_{p}(u)} \text {. }
$$

(cf. (2)), where the differentials on the left correspond to the differentials obtained on the right by alternating sums from the cofaces of the co- 
simplicial object $I^{N(\vartheta)}$. We claim that $C^{p}\left(U, \varphi^{*} I\right)$ is an acyclic complex. Since $I$ is injective, it suffices to prove that Free $(N . U)$ is an acyclic chain complex in $\underline{\mathrm{Ab}}(\mathscr{E})$, where Free $(-)$ denotes the free abelian group functor. To this end, let $p: \mathscr{B} \rightarrow \mathscr{E}$ be a Boolean extension as at the end of Section 1 , and consider the pullback square

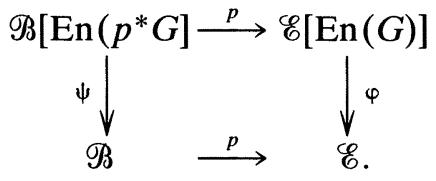

Since $\varphi$ is locally connected so is $\psi$, and the Beck-Chevalley condition holds, i.e.

$$
p^{*} \varphi_{!} \cong \psi ! p^{*}
$$

Consequently, if we write $u^{\prime}$ for the cover of $\operatorname{En}\left(p^{*} G\right)$ induced by $U$ via pullback along $p$, we have $p^{*}(\operatorname{Free}(N . \mathcal{U})) \cong \operatorname{Free}\left(N . U^{\prime}\right)$. But $\mathscr{B}$ is a model for set theory (with the axiom of choice), so we are now in a position to apply results from classical topology: the cover $u^{\prime}$ of $\operatorname{En}\left(p^{*} G\right)$ is a cover by basic opens, and $\operatorname{En}\left(p^{*} G\right)$ as well as each of its basic open subspaces are contractible, so the nerve $N\left(U^{\prime}\right)$ of this cover is a contractible simplicial set, and Free $\left(N U^{\prime}\right)$ is an acyclic chain complex. Since $p^{*}($ Free $N u)=\operatorname{Free}\left(N u^{\prime}\right)$ and $p^{*}$ is faithful, it follows that Free $(N U)$ is acyclic, as was to be shown.

Now apply this argument not just to $\operatorname{En}(G)$, but to any basic open $B \subset e_{E}^{*}(\operatorname{En}(G))$ and any $E \in \mathscr{E}(\operatorname{cf}(11)$, where $Y=\operatorname{En}(G)$ now). Then we conclude that $\mathbf{B}$ is an $I$-acyclic basis. (12) now follows by Lemma 2 , since the whole space $\operatorname{En}(G)$ is a member of $\mathbf{B}$. This completes the proof of the theorem.

5. Torsors. Let $G$ be a group in a topos $\mathscr{E}$. A $G$-torsor in $\mathscr{E}$ (or principal $G$-bundle over $\mathscr{E}$ ) is an object $T$ of $\mathscr{E}$ equipped with an action $\mu: G \times T \rightarrow T$ of $G$ such that $T \rightarrow 1$ is epi and $\left(\mu, \pi_{2}\right): G \times T \rightarrow$ $T \times T$ is an isomorphism. Recall ([Gi]) that $H^{1}(\mathscr{E}, G)$ is the pointed set of isomorphism classes of $G$-torsors (this is a group if $G$ is abelian). For a space $X$ and a sheaf of groups $G$ on $X, H^{1}(X, G)$ stands for $H^{1}(\operatorname{Sh}(X), G)$. 
Theorem. Let $\mathscr{E}$ be a topos, and let $\varphi: \operatorname{Sh}\left(X_{\mathscr{E}}\right) \rightarrow \mathscr{E}$ be the cover of Section 1. For any group $G$ in $\mathscr{E}, \varphi$ induces an isomorphism

$$
H^{1}(\mathscr{E}, G) \stackrel{\sim}{\rightarrow} H^{1}\left(X_{\mathscr{E}}, \varphi^{*} G\right)
$$

Proof. The functor $\varphi^{*}: \mathscr{E} \rightarrow \operatorname{Sh}\left(X_{\mathscr{E}}\right)$ is fully faithful, so it restricts to a fully faithful functor from the category of $G$-torsors in $\mathscr{E}$ to that of $\varphi^{*} G$-torsors in $\operatorname{Sh}\left(X_{\mathscr{E}}\right)$. It thus suffices to show that this restriction of $\varphi^{*}$ is essentially surjective. By $[\mathrm{JM}]$, there is a class $P \subset\left(X_{\mathscr{E}}\right)^{I}$ of paths, such that $\mathscr{E}$ is equivalent to the full subcategory of $\operatorname{Sh}\left(X_{\mathscr{E}}\right)$ consisting of those sheaves on $X_{\mathscr{E}}$ which are constant along the paths in $P$. Let $T$ be a $\varphi^{*} G$-torsor in $\operatorname{Sh}\left(X_{\mathscr{E}}\right)$. Then $T$ is locally isomorphic to $\varphi^{*}(G)$, and $\varphi^{*}(G)$ is constant along all the paths in $P$. So $T$ is locally constant along the paths in $P$, and hence constant along those paths (since the interval $I$ is simply connected).

6. Etale homotopy. Let $\mathscr{E}$ be a locally connected topos, and let $p$ be a point of $\mathscr{E}$. Artin and Mazur ([AM]) define the etale homotopy groups $\pi_{n}(\mathscr{E}, p)(n \geq 0)$, and prove a Whitehead theorem for toposes: a geometric morphism $(\mathscr{F}, q) \rightarrow(\mathscr{E}, p)$ of pointed locally connected toposes induces isomorphisms of etale homotopy groups iff it induces isomorphisms of cohomology groups with coefficients in a locally constant abelian group $A$ in $\mathscr{E}$, as well as an isomorphism of the fundamental progroups $\pi_{1}(\mathscr{F}, q) \rightarrow \pi_{1}(\mathscr{E}, p)$. Our previous results give:

Corollary. For any locally connected pointed topos $(\mathscr{E}, p)$ there exists a pointed space $\left(X_{\mathscr{E}}, q\right)$ and a cover $\varphi:\left(\operatorname{Sh}\left(X_{\mathscr{E}}\right), q\right) \rightarrow(\mathscr{E}, p)$ which induces isomorphisms in etale homotopy,

$$
\pi_{n}\left(X_{\mathscr{E}}, q\right) \stackrel{\sim}{\rightarrow} \pi_{n}(\mathscr{E}, p) \quad(n \geq 0)
$$

Proof. First of all, we need to modify the construction of the space $X_{\mathscr{G}}$ slightly, in order to lift the point $p$ : if we replace the set $\mathbf{N}$ of natural numbers by an arbitrary infinite set $S$ in the construction of Section 1 (and the space of infinite-to-one enumerations $\mathbf{N}<U \rightarrow G$ by that of infinite-to-one partial maps $\Delta(S)<-U \longrightarrow G$, where $\Delta S$ denotes the constant object of $\mathscr{E}$ corresponding to the set $S$ ), we obtain a cover (again called) $\varphi: X_{\mathscr{E}} \rightarrow \mathscr{E}$ with exactly the same properties as before. A straightforward classifying-topos argument shows that if we 
choose the cardinality of $S$ sufficiently large (at least that of $p^{*} G$ ) then the given point $p$ can be lifted to a point $q$ of this (modified) space $X_{\mathscr{\&}}$. $X_{\mathscr{E}}$ is locally connected since $\mathscr{E}$ is, and $\varphi$ is a locally connected map. Now the result of Section 5 shows that $\varphi$ induces an isomorphism in $\pi_{1}$ (since $H^{1}(\mathscr{E}, G) \cong \operatorname{Hom}\left(\pi_{1}(\mathscr{E}, p), G\right)$, cf $[\mathrm{AM}]$, Section 10). The corollary follows by the Whitehead theorem just quoted and the theorem of Section 4.

UNIVERSITÉ DU QUÉBEC À MONTREAL, CANADA

UNIVERSITY OF CHICAGO

REFERENCES

[AM] M. Artin and B. Mazur, Etale Homotopy, Springer LNM, 100 (1969).

$\rightarrow$ M. Barr, Toposes without points, J. Pure and Applied Alg., 5 (1974), 265-280.

$[\rightarrow$ A. Boileau and A. Joyal, La logique des topos, J. Symb. Logic, 46 (1981), 6-16.

[CE] H. Cartan and S. Eilenberg, Homological Algebra, Princeton (1956).

[Gi] J. Giraud, Cohomologie Non-Abélienne, Springer Verlag (1971).

[G] A. Grothendieck, Sur quelques points d'algèbre homologique, Tohokû Math. J., 9 (1957), 119-221.

[J] P. T. Johnstone, Topos Theory, Academic Press (1977).

[JM] A. Joyal and I. Moerdijk, Toposes as homotopy groupoids, (to appear in Advances in Math.).

[JT] A. Joyal and M. Tierney, An extension of the Galois theory of Grothendieck, Memoirs AMS, 309 (1984).

[V] J.-L. Verdier, Cohomologie dans les topos, SGA 4, exposé V, Springer LNM, 270 (1972). 